\title{
Flow stress models for deformation under varying condition-finite element method simulation
}

\author{
Dmytro Svyetlichnyy $^{1} \cdot$ Jaroslaw Nowak $^{1} \cdot$ Nikolay Biba $^{2} \cdot{\text { Lukasz } \text { Lach }^{1}}^{1}$
}

Received: 15 October 2015 / Accepted: 9 February 2016 / Published online: 24 February 2016

(C) The Author(s) 2016. This article is published with open access at Springerlink.com

\begin{abstract}
This work presents description and comparison of internal and state variable models of flow stress in varying processing conditions. Three models were analyzed. The first one is based on dislocation theory and describing the mechanical behavior of f.c.c. polycrystalline structures. The second and third models are standard and modified Sellars' flow stress models. Models were adapted for two commercial codes based on finite element method: QForm7 and Forge 2005. The compression test of 45 grade steel with instant changes of strain rate was simulated. Calculated compression force and flow stress were compared with the experimental data from plastometric tests. The forging process was simulated by QForm7. Results obtained by both internal and modified Sellars' models confirm their high accuracy for analysis and prediction of the flow stress under the varying deformation conditions.
\end{abstract}

Keywords Flow stress · Internal variables model · Varying deformation conditions $\cdot$ FEM simulation

\section{Introduction}

A proper description of the flow stress under varying conditions is particularly beneficial for computer simulation because the real processing conditions change continuously.

Łukasz Łach

lach@agh.edu.pl

1 AGH University of Science and Technology, Krakow, Poland

2 MICAS Simulations Ltd., Oxford, UK
For example, the strain rate usually grows at the beginning of the process, then reaches a maximum value, maintains it for a certain period at approximately the same level, and finally decreases to zero at the end of the process. Furthermore, the deformation temperature does not remain constant. These changes of the deformation conditions occur constantly in various areas of the deformed body and with different intensity. It all leads to the conclusion that only those models that describe the real mechanical behavior of material under varying deformation conditions guarantee precise assessment and are suitable for computer simulation.

Most existing flow stress models, describing mechanical response of the deformed body, treat the deformation as a stationary process. Some of them consider only the current values of deformation parameters (strain, strain rate, temperature), and they are referred to as state variable models (SVM). Other models take into account the history of deformation, describe the internal state of the material, and use internal variables. Time is included into these models explicitly or implicitly, and these models are known as internal variables models.

For the SVMs, it is not critical in what way the strain rate or temperature is changing during the deformation; the determining factor is only the current values. Therefore, in SVMs, variations of deformation conditions lead to the instant changes of the flow stress.

The internal variables models (IVM) describe the flow stress as a continuous transient process, i.e., from the initial state to the final state. The final state is a stationary deformation process with a constant strain rate at a constant temperature and flow stress. There are well-known models developed by Kocks [1], Roberts [2], Yoshie et al. [3], Bergstrom [4], and Estrin and Mecking [5], which use the dislocation density as an internal variable. These models are said to have an advantage when a non-stationary process takes place. 
Kocks and Mecking [6] have shown that in most cases, one internal variable is sufficient to describe the flow stress for materials with the f.c.c. structure in the wide range of the strain rate and temperature. However, they also stated, that one internal variable allows to describe only a process with constant deformation conditions. Estrin et al. [7], Roters et al. [8], and van Houtte [9] came to the similar conclusion and proposed to introduce additional internal variables. Sandström and Langeborg [10] suggested using the distribution function instead of one value of the dislocation density. However, the main objective of the variable addition was not to take into account the varying deformation conditions, but the necessity of considering certain specific conditions. Introduction of the additional variables is connected with a more precise description of the deformation with considerable strain, changes of the deformation path, evolution of the dislocation structure, and texture or recrystallization.

The non-stationary deformation processes were considered on the basis of the IVM for example by Routcoueles et al. [11] and Ordon et al. [12]. These authors declared satisfactory results, but one cannot recognize them as appropriate enough.

The IVMs are represented by a differential equation or a system of two or three differential equations. In addition, they can be expanded by an independent equation of dislocation structures evolution, which is essentially a solution of another differential equation. All the models presented above [1-5, 7-12] can be classified as additive models, because the effect of almost every element can be considered as an additional term of the sum.

However, multiplicative models have also been developed. Kocks and Mecking [6] argued that every transient is evidence for an internal state parameter that evolves towards its steady state under the given applied conditions. The existence of a transient upon a change in externally prescribed conditions calls for an additional internal state parameter. Kocks and Mecking [6] have given a physical explanation and have offered a way to resolve the problem of varying deformation conditions. Another solution was proposed by Estrin [13]. The two-internal-variable formulation was devised for this purpose. A more fundamental proposition was to consider them not additively, but multiplicatively.

Another multiplicative IVM was developed [14] and validated [15] by Svyetlichnyy in order to take into account the recrystallization process. The model demonstrates exceptional ability for a proper description evolution of the dislocation density not only during the deformation but also after it. Later, Svyetlichnyy et al. [16] extend the multiplicative model on varying deformation conditions. In the paper [16], the results of experimental studies and theoretical analysis clearly show that the rheological model should be multiplicative. It is more important than the choice between one or more internal variable. A good prediction was achieved using multiplicative model for the analysis of the flow stresses of hot-compressed
45 grade steel. Model parameters were identified and verified based on the data of plastometric tests.

Presented paper is the continuation of the previous work. An objective is a model validation by finite element method (FEM) simulation. For this purpose, models were implemented into two commercial FEM codes, and plastometric tests were simulated.

\section{Flow stress models}

Three flow stress models were analyzed in the study. The first one is an internal variable model [16] based on dislocation theory and describing the mechanical behavior of f.c.c. polycrystalline structures. The second and third models are standard and modified Sellars' flow stress models.

\subsection{Internal variable model}

The model [16] is based on Taylor's dislocation theory [17]. In the model, the following equation describes the flow stress as a function of the general internal variable $\rho_{a v}$ :

$\sigma=\sigma_{0}+\alpha \mu b \sqrt{\rho_{a v}}$

where: $\sigma_{0}$ - stress necessary to move the dislocation in the absence of other dislocations, $\alpha$ - constant, $\mu$ - shear modulus, $b$-magnitude of the Burgers vector, and $\rho_{a v}$ - general internal variable (average dislocation density).

The general internal variable $\rho_{a v}$ is a product of three multipliers:

$\rho_{a v}=k_{\rho} \rho_{m}(1-\chi)$

where: $k_{\rho}$-factor, which takes into account the real deformation condition, $\rho_{m}$-normalized dislocation density, and $\chi-$ fraction volume of the recrystallized grains.

Normalized dislocation density $\rho_{m}$ can be calculated by using the following equation [16]:

$\dot{\rho}_{m}=u \dot{\varepsilon}-u \rho_{m} \dot{\varepsilon}-r \rho_{m}$

where: $u$ and $r$ are the parameters, which depend on material; $u$ is responsible for hardening and dynamic recovery, $r$-for static recovery. Equation (3) is discussed in detail elsewhere [16].

Factor $k_{\rho}$ is responsible for implementation into the model the real deformation conditions. Factor $k_{\rho}$ is a function of the temperature $T$ and the strain rate $\dot{\varepsilon}$, and then it can be determined through the ZenerHollomon parameter $Z=\dot{\varepsilon} \exp \left(\frac{Q}{R T}\right)$. When the deformation conditions are changed, factor $k_{\rho}$ is changed as well. Factor $k_{\rho}$ does not change its value instantly, but some deformation is required for transient process. 
Therefore, factor $k_{\rho}$ can be described by the following differential equation:

$\varepsilon_{v} \frac{d k_{\rho}}{d \varepsilon}+k_{\rho}=A Z^{n}$

where: $\varepsilon_{v}$-characteristic strain for varying conditions.

Fraction of recrystallization $\chi$ is calculated via extended volume $V_{e x}$ :

$\chi=1-\exp \left(-V_{e x}\right)$

Model proposed by Sellars [18] or cellular automata [19-22] could be used for calculation of the fraction of recrystallization $\chi$. But it is calculated in the way proposed by Svyetlichnyy [14]. Four differential state equations are used for the extended volume $V_{e x}$ calculations. Thus, number of grains $N_{v}$ extended size $D_{e x}$, and area $S_{e x}$ of the grains should be calculated:

$\dot{N}_{V}=v_{N}\left[1-\frac{N_{V}}{N_{\max }}\right]$

$\dot{D}_{e x}=v\left[N_{V}-\frac{D_{e x}}{D_{\max }}\right]$

$\dot{S}_{e x}=\frac{v}{2}\left[\frac{\pi}{4} D_{e x}-\frac{S_{e x}}{D_{\max }}\right]$

$\dot{V}_{e x}=\frac{v}{3}\left[\frac{2}{3} S_{e x}-\frac{V_{e x}}{D_{\max }}\right]$

where: $v_{N}$-nucleation rate, $v$-grain growth rate, $N_{\max }$-maximal number of grains in volume unit, and $D_{\max }$-maximal extended size of the grains.

\subsection{State variable model (SVM)}

The model developed by Davenport et al. [23], which is based on Sellars and Tegart concept [24], is taken as an example of such a SVM. The model is described by the following equation:

$\sigma=\sigma_{0}+\left(\sigma_{s}-\sigma_{0}\right)\left[1-\exp \left(-\frac{\varepsilon}{\varepsilon_{r}}\right)\right]^{\frac{1}{2}}-R_{x}$

where: $\sigma_{0}, \sigma_{s}$-initial and saturated value of flow stress, $\varepsilon_{r}-$ characteristic strain, and $R_{x}$ - term, which takes into account dynamic recrystallization. All these parameters of the materials $\left(\sigma_{0}, \sigma_{s}, \varepsilon_{r}\right.$, and $\left.R_{x}\right)$ are functions of the strain rate and temperatures. This model is described in details elsewhere [23].

Recrystallization is taken into account according to the following expression:

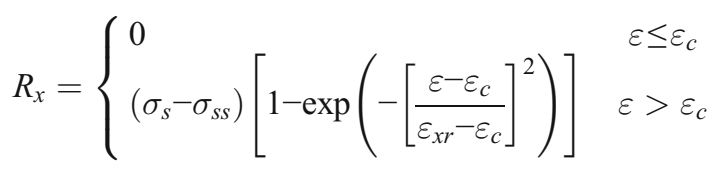

where $\varepsilon_{c}-$ critical strain:

$\varepsilon_{c}=C_{c}\left(\frac{Z}{\sigma_{s}^{2}}\right)^{N_{c}}$,

$Z$-Zener-Hollomon parameter with activation energy $Q$.

The other parameters are as following:

$$
\begin{aligned}
\sigma_{0} & =\frac{1}{\alpha_{0}} \sinh ^{-1}\left(\frac{Z}{A_{0}}\right)^{\frac{1}{n_{0}}}, \sigma_{s}=\frac{1}{\alpha_{s}} \sinh ^{-1}\left(\frac{Z}{A_{s}}\right)^{\frac{1}{n_{s}}}, \sigma_{s s} \\
& =\frac{1}{\alpha_{s s}} \sinh ^{-1}\left(\frac{Z}{A_{s s}}\right)^{\frac{1}{n_{s s}}}, \varepsilon_{r}=\frac{q_{1}+q_{2} \sigma_{s}^{2}}{3.23}, \varepsilon_{x r}-\varepsilon_{c} \\
& =\frac{\varepsilon_{x s}-\varepsilon_{c}}{1.98}, \varepsilon_{x s}-\varepsilon_{c}=C_{x}\left(\frac{Z}{\sigma_{s}^{2}}\right)^{N x}
\end{aligned}
$$

\subsection{Modified state variable model (mSVM)}

To rebuild the SVM, in order to consider varying conditions, it is separated into two parts according to the papers [16]. The first one is independent or almost independent from deformation conditions (term in square brackets of Eq. (10)) and depends on the strain and characteristic strain, which may change in a narrow range. Another part introduces deformation conditions and depends on the strain rate and temperature. It consists of parameters $\sigma_{0}, \sigma_{s}$, and $R_{x}$ in Eq. (10). Deformation conditions apply to them. In the original model Eq. (10), the parameters are changed instantly according to current conditions without lag or delay. For proper accounting of the varying conditions, the parameters should be changed not instantly, but smoothly.

Then, the three equations are added to the model. They are of the following form:

$$
\begin{aligned}
& \varepsilon_{v} \frac{d \sigma_{0}}{d \varepsilon}+\sigma_{0}=\sigma_{0 c} \\
& \varepsilon_{v} \frac{d \sigma_{s}}{d \varepsilon}+\sigma_{s}=\sigma_{s c} \\
& \varepsilon_{v} \frac{d R_{x}}{d \varepsilon}+R_{x}=R_{x c}
\end{aligned}
$$

where index $c$ defines parameters calculated according to Eqs. (11)-(13), parameters without index $c$ consider varied conditions, and they are to be substituted into Eq. (10); and $\varepsilon_{v}$ - characteristic strain, the same for all the parameters.

Such a modification introduces into the SVM three internal variables. Therefore, modified state variable model (mSVM) receives properties of IVM.

\subsection{Models parameters for carbon steel of grade 45}

Basic chemical composition of steel 45 is shown in Table 1. Plastometric tests were carried out by using the Gleeble 3800 
Table 1 Chemical composition of the steel 45 in wt. \%

\begin{tabular}{lllll}
\hline $\mathrm{C}$ & $\mathrm{Mn}$ & $\mathrm{Si}$ & $\mathrm{P}, \mathrm{S}$ & $\mathrm{Cr}, \mathrm{Ni}, \mathrm{Cu}$ \\
\hline 0.45 & 0.6 & 0.2 & $<0.04$ & $<0.3$ \\
\hline
\end{tabular}

thermomechanical simulator under constant and varying deformation conditions. Conditions and results of the plastometric tests can be found in previous paper [16]. Based on experimental data, parameters of the three models were identified.

For IVM Eqs. (1-9), nucleation rate $v_{N}$, grain growth rate $v$, maximal number of grains in unit volume $N_{\max }$, maximal extended size of the grains $D_{\max }$, and other parameters can be defined as following. For stress calculation $\sigma$ Eq. (1):

$$
\begin{gathered}
\sigma_{0}=9.29 \dot{\varepsilon}^{0.242} \exp \left(\frac{8700}{\mathrm{RT}}\right), \alpha=1, \mu=7.5 \cdot 10^{10}, \\
b=2.58 \cdot 10^{-10} .
\end{gathered}
$$

For calculation of normalized dislocation density $\rho_{m}$ Eq. (3):

$u=1, r=6410 \exp \left(-\frac{127500}{\mathrm{RT}}\right)$.

For calculation of factor $k_{\rho}$ Eq. (4):

$\varepsilon_{v}=0.0179, Z=\dot{\exp }\left(\frac{486700}{\mathrm{RT}}\right), A=0.0291, n=0.154$.

For calculation of the number of grains $N_{v}$ Eq. (6), extended size $D_{e x}$ Eq. (7), area $S_{e x}$ Eq. (8) and volume $V_{e x}$ Eq. (9):

$$
\begin{gathered}
v_{N}=1.516 \cdot 10^{8}, v=3.638 \cdot 10^{9} \exp \left(-\frac{283000}{\mathrm{RT}}\right), \\
N_{\max }=5.86 \cdot 10^{-6}\left[\dot{\exp }\left(\frac{300000}{\mathrm{RT}}\right)\right]^{0.81}, \\
D_{\max }=16.48\left[\exp \left(\frac{292000}{\mathrm{RT}}\right)\right]^{-0.225} .
\end{gathered}
$$

All parameters of the SVM and mSVM are collected in Table 2

\section{Models implementation into FEM codes-verification}

For models verification, they were implemented into the three FEM codes: QForm7®, Forge 2005, and a code developed in AGH (Poland) by Malinowski [25, 26]; and after simulation, results were compared with experimental data and other models. Each FEM code allows the possibility for implementation of users' function for flow stress. It should be a Fortran
Table 2 Parameters of the SVM and $\mathrm{mSVM}$

\begin{tabular}{lll}
\hline Parameter & SVM & $\mathrm{mSVM}$ \\
\hline$Q$ & 313400 & 309400 \\
$\alpha_{0}$ & 0.0166 & 0.0233 \\
$A_{0}$ & $9.955 \times 10^{19}$ & $1.687 \times 10^{19}$ \\
$n_{0}$ & 10.0 & 14.58 \\
$\alpha_{s S}$ & 0.00516 & 0.00437 \\
$A_{S S}$ & $7.393 \times 10^{14}$ & $1.356 \times 10^{14}$ \\
$n_{S S}$ & 4.24 & 4.59 \\
$\alpha_{s}$ & 0.00491 & 0.00480 \\
$A_{s}$ & $1.536 \times 10^{14}$ & $9.527 \times 10^{13}$ \\
$n_{s}$ & 7.02 & 7.15 \\
$C_{c}$ & 0.0617 & 0.0336 \\
$N_{c}$ & 0.0518 & 0.0839 \\
$C_{x}$ & 0.00610 & 0.00682 \\
$N_{x}$ & 0.239 & 0.236 \\
$q_{1}$ & 0.340 & 0.407 \\
$q_{2}$ & $10^{-7}$ & $10^{-7}$ \\
$\varepsilon_{v}$ & - & 0.0144 \\
\hline
\end{tabular}

subroutine for Malinowski's code. Fortran subroutines were also used for model implementation into Forge 2005, while experimental data in table form are introduced in that code for comparison. The flow stress could be presented in QForm7® as a table, function, or LUA subroutine.

Plastometric compression test was the process in every simulation variant. Experimental data were obtained by using the Gleeble 3800 thermomechanical simulator. Description of the principles of experimental method of hot compression test with used Gleeble can be found elsewhere [27]. Cylindrical specimens with diameter of $10 \mathrm{~mm}$ and height of $12 \mathrm{~mm}$ were compressed under different conditions, which are described in detail in [16]. Two simulation series the same as real plastometric tests were fulfilled. The first series were carried out in constant deformation conditions at three different temperatures $\left(800,900\right.$, and $\left.1000{ }^{\circ} \mathrm{C}\right)$ and three different strain rates $\left(0.1,1.0\right.$, and $\left.10 \mathrm{~s}^{-1}\right)$. The second series were done under varying deformation condition at constant temperature and instant changes of strain rate at different strain.

Firstly, simulations were carried out with the Malinowski's code. Simulation results of two models (IVM and Shida's [28] model) were compared with flow stress and compression force obtained in plastometric tests. IVM gave better results because parameters of IVM were identified exactly for this material. Those results are not presented in this paper in view of very limited application of Malinowski's code. More attention is paid to the commercial codes Forge 2005 and QForm 7.

The next numerical simulations of uniaxial compression test for steel of grade 45 was performed with use of commercial FE software Forge 2005 as a three-dimensional simulation. Specimen was thermally isolated from dies and air 
cooled on free surface. Process parameters are as follows: specimen height $h=12 \mathrm{~mm}$, diameter $d=10 \mathrm{~mm}$, die stroke $\Delta h=8 \mathrm{~mm}$, and friction coefficient $\mu=0.25$. The friction law is based on the combined Coulomb and Treska friction laws. Heat transfer coefficient was selected from the Forge database and depends on temperature. The friction coefficient, friction factor, and heat transfer coefficient were given as input data in Forge for the process calculations (the values are defined in the input file).

Two variants of flow stress were compared. The first one is the table representation. The table contains flow stress for different temperature, strain rate, and strain; commonly, there are several stress-strain curves for different deformation conditions. Nonlinear interpolation is used when deformation conditions are not equal to the table values. For simulation presented in the paper, the curves obtained by experimental tests on Gleeble were written into the table. It is the most effective kind of SVM, especially for the standard conditions. The second variant of flow stress is a user subroutine according to the developed IVM (section 2.1) with parameters presented in section 2.4 (Table 2).

Some examples of temperature and strain distribution on the surface and cross-section of the specimen are presented in Fig. 1. Examples of compression forces measured and calculated by two methods are presented in Figs. 2 and 3. Table representation of flow stress with nonlinear interpolation is marked as "interpolation;" results obtained with use IVM subroutine is marked as "calculated." Simulation results for variants under the constant deformation condition (Fig. 2) do not show significant differences with small advantage of table form of flow stress. Actually, overall error is the sum of errors appearing in all sequences from measured to calculated force. This sequence is shorter in the case of the table than for the analytical model. Some inaccuracy is connected with measurements, others with the process and natural variations of material properties. In addition to this, there are some sources of common errors of these two methods. The first error appears in calculation of flow stress on the data of compressive force and strain on the data of displacements. It depends on accuracy of consideration of deformation conditions. Here, we rely on program code delivered by Dynamic Systems Inc. with the thermomechanical simulator Gleeble-3800. Then, strain-stress curves are smoothed by data filtering, and strain rate is calculated so that only 30 to 100 points remain on each curve for use in the table or in the models. This eliminates errors of high frequency and smooth oscillations appearing in equipment during deformation with high strain rate (over $20 \mathrm{~s}^{-1}$ ). Effect of smoothing can be observed in Fig. 2b. Next common error connects with conditions of simulation: heat transfer, torque, tool velocity, and other.

An additional error in the table method depends on interpolation of the deformation conditions that may be different from those written in the table (different temperature, strain, strain rate). That error is very small because the modeling process is simulated in a condition very close to the condition for which table data were received. When models are used for flow stress calculations, two additional errors appear. The first one depends on model properties, i.e., model adequacy, ability for proper description, and consideration factors effecting on the final results. The second error is the model identification error appearing when model parameters are defined with insufficient accuracy. Thus, for the same test conditions as in experimental study, the table representation should provide the same values of the flow stress and consequently compressive force. Such coincidence is guaranteed because the simulation is based on interpolation of experimental data. Using any kind of material models gives us the results less (Fig. 2a) or more (Fig. 2b) close to experiment because the models provide approximation, but not interpolation of experimental data.

Other results are obtained when deformation conditions are changed. Then, IVM reflects force better than the table (Fig. 3). Instant changes of strain rate lead to instant drop of compression force when the table is used for flow stress calculation, while force calculated by IVM demonstrates smooth changes that are more close to the measured force. It shows advantage of IVM for varying deformation condition in comparison with the most effective SVM (i.e., table with interpolation), which could not consider varying deformation conditions. Therefore, IVM is more effective for such conditions.

Three models described in previous section were studied by simulation by commercial FEM code QForm7, and results are presented in this section. Finite element code QForm 7 provides simulation of large deformation either in rigidplastic or in elastic-visco-plastic formulation using triangle (2D) of tetrahedral (3D) elements. Deformation of the tools can be calculated concurrently with the material flow as a coupled problem. More details can be found elsewhere [29, 30]. Simple functions for flow stress were applied as well but these functions gave significantly worse results. The table interpolation method demonstrates very close results to the ones obtained by Forge 2005 for the same conditions.

In the case of models comparison, all errors connected with flow stress, strain and strain rate calculation, data filtering, models parameters identifications, and simulation conditions are the same. Only errors connected with the model's structure and properties are different, and this difference was analyzed in paper [16], where flow stress was compared for three models. It is analyzed below.

Some results are presented in Fig. 4. Simulation results of the first variant with linear decrease of the strain rate from 1 to $0.1 \mathrm{~s}^{-1}$ at the strain 0.01 are presented in Fig. 4a. Here, the force calculated by the IVM is very close to the real forces obtained in plastometric tests. The force calculated by SVM drops much faster. Transition period can be seen in real process and simulation with IVM also when strain rate increases (Fig. 4b). 

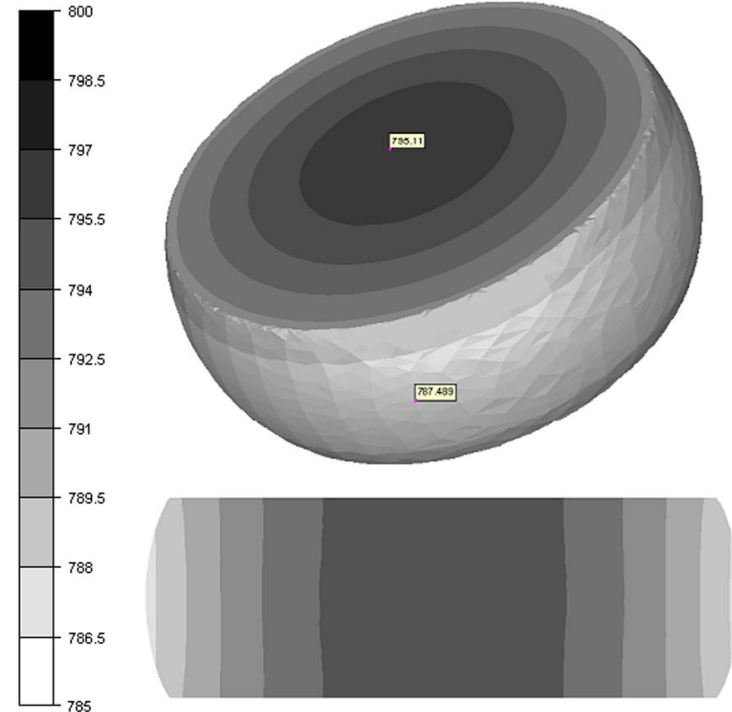

(a)
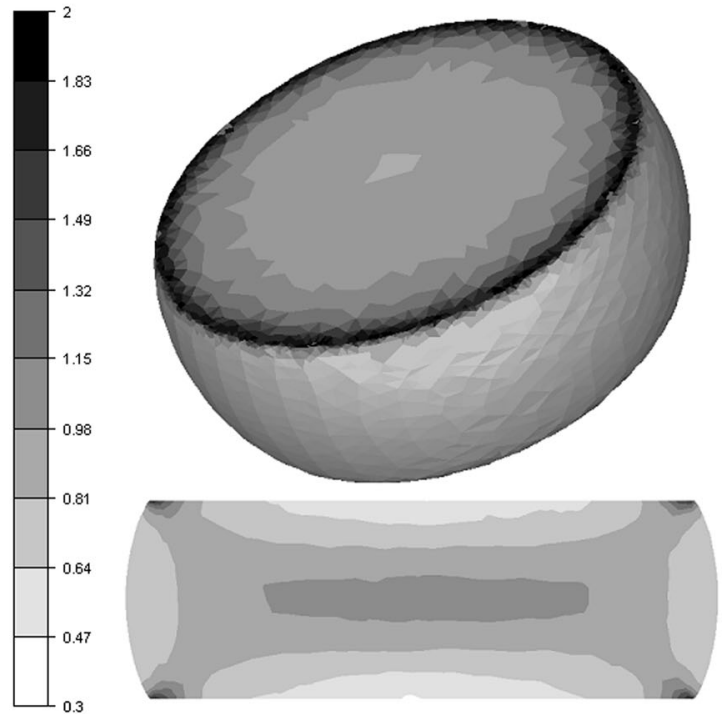

(b)

Fig. 1 Temperature $\left[{ }^{\circ} \mathrm{C}\right](\mathbf{a})$ and equivalent strain (b) distribution after the deformation at $800{ }^{\circ} \mathrm{C}$ and strain rate $0.1 \mathrm{~s}^{-1}$

The modified model (mSVM) is tested on the same experimental data. Examples of a comparison SVM and mSVM are presented in Fig. 5. Flow stresses for a central point of the specimen from experimental data are presented by the lines with symbols, SVM curves are dashed lines, while the results of the mSVM are shown by continues lines. The modified model gives results which are closer to the experiments than the original model. The weighted average normalized error equals $3.2 \%$ for original SVM and $2.55 \%$ for $\mathrm{mSVM}$, which is very close to the error $(2.41 \%)$ for IVM. Though, the weighted average normalized error is calculated for all curves with constant and varying condition, as well as transition period is only a small part of the entire process. This error does not fully reflect the difference in the transition process.
The results of FEM simulation by QForm7 show the advantage of IVM (and partly $\mathrm{mSVM}$ ) for varying deformation condition in comparison with simple SVM, which could not consider such conditions. Therefore, IVM is more effective for varying conditions.

\section{Forging process modeling}

Analysis of model behavior in the simple process of axial compression test gives some difference only during short transient time when strain rate is changed. When deformation conditions are constant, the differences in simulation results
Fig. 2 Measured and calculated forces: (a) temperature $800{ }^{\circ} \mathrm{C}$, strain rate $10 \mathrm{~s}^{-1} ;(\mathbf{b})$ temperature $900{ }^{\circ} \mathrm{C}$, strain rate $100 \mathrm{~s}^{-1}$

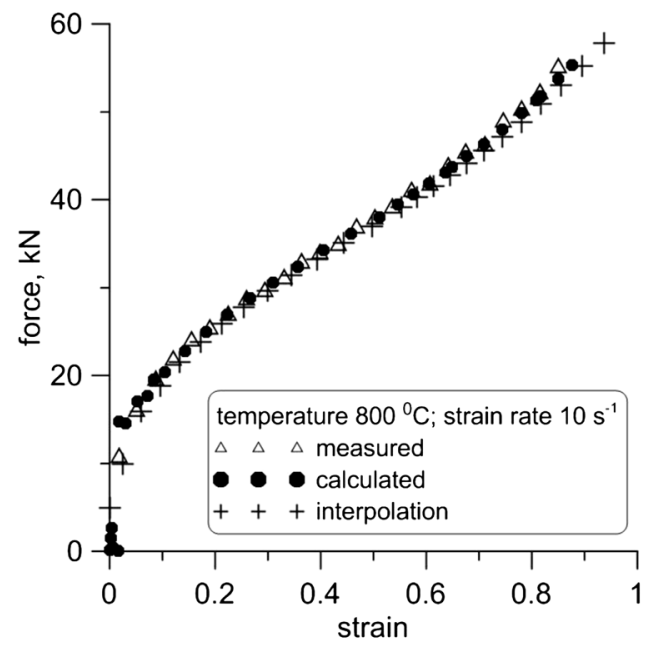

(a)

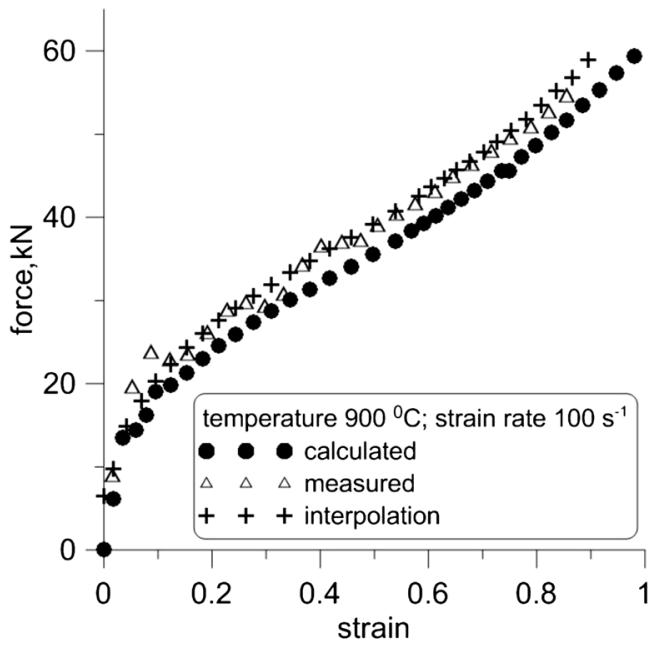

(b) 


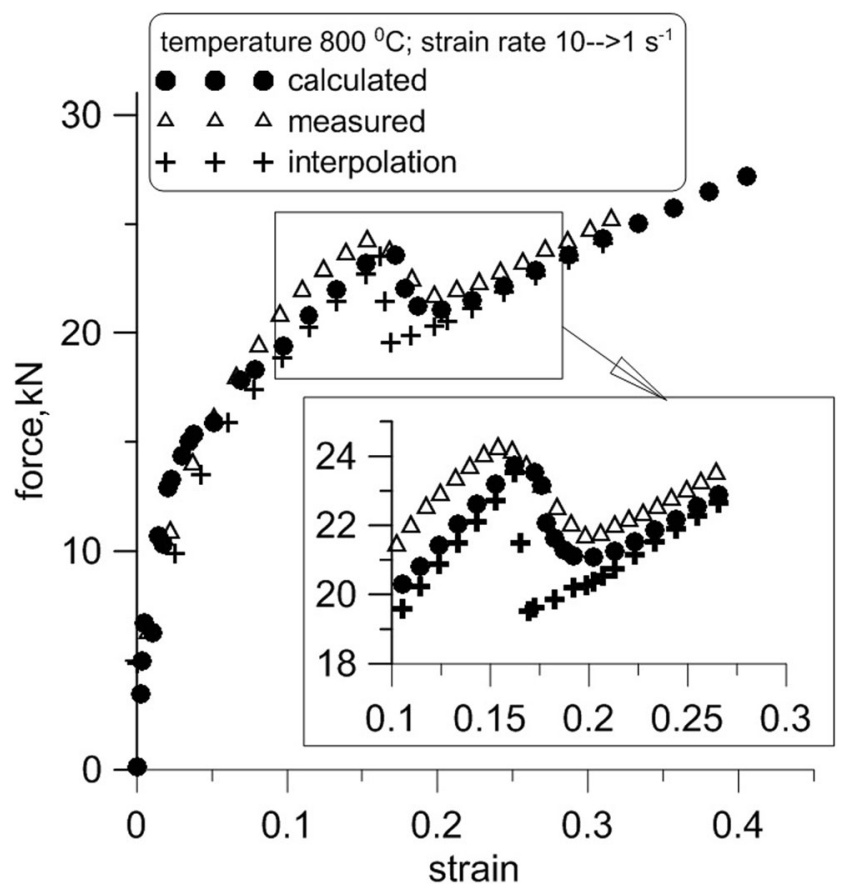

Fig. 3 Measured and calculated force during the deformation at the temperature of $800{ }^{\circ} \mathrm{C}$ with decreasing strain rate

by different models cannot be observed at all. The compression test is carried out in an idealized way, which provides maximal possible uniform deformation. It allows for obtaining reliable results for model identification, but real processes differ by non-uniformed deformation and varying conditions. Changes in different locations during the processes occur not simultaneously. Processes more complex than axial compression test must be simulated to determine how significant the effect of varying conditions can be.

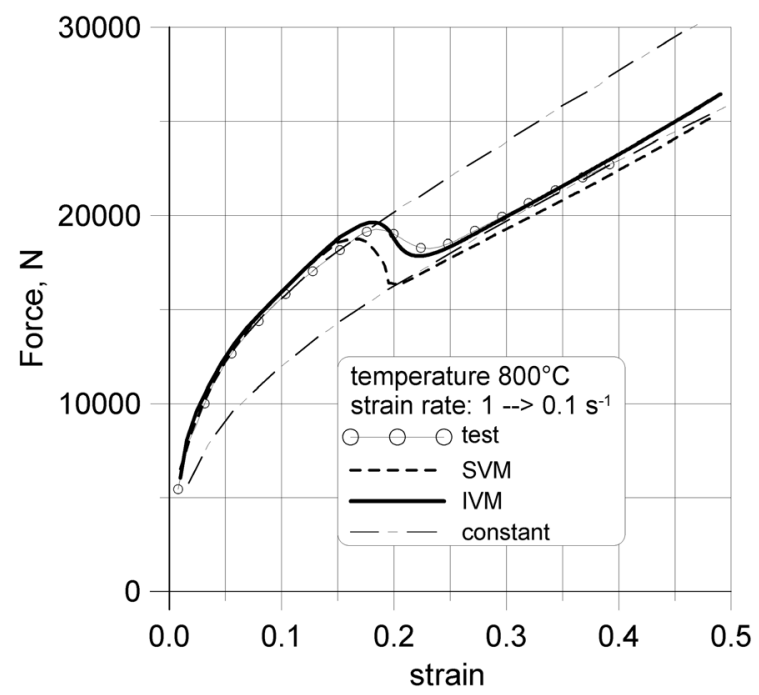

(a)
Forging process on $85 \mathrm{MN}$ press was chosen as an example for analysis of models behavior. FEM simulations were carried out with use of QForm7. Cylindrical workpiece was forged in closed dies of a complex shape. Axisymmetric process allows applying two-dimensional calculations. Initial temperature of workpiece was $1200{ }^{\circ} \mathrm{C}$. Simulation results obtained with use of IVM, SVM, and mSVM described above are presented in Figs. 6, 7, and 8. Effective stress distributions for five moments of the one-stage forging process are shown in Fig. 6. The modeled process is characterized by a very high non-uniform deformation and, as a result, non-uniform stress. Here, five points located in different places are presented as well. Changes of flow stress in three points during the forging process are drawn in Figs. 7 and 8. The highest stress is observed for the point located at the bottom of piece, which come into contact with the bottom tool at the beginning of the process, while the lowest stress for almost the entire process remained in the center of the piece, near the axis.

Forging force obtained by the three models is almost the same for entire process (Fig. 6); some difference can be observed only at the end of the process. However, significant differences can be seen in effective stress distribution. No control points demonstrate the same changes of the stress for the different models. Sometimes, the differences are small and last only for a short time. For example (Fig. 8a), at the point 1, less stress is obtained for SVM model at the end of the process. At the same time, at point 0 , stress for $\mathrm{mSVM}$ remains at the same level, while for SVM, two steps in stress can be observed. The most difference was seen at point 3 (Fig. 8b) at the bottom part of the workpiece near the bottom tool. SVM demonstrates significant decrease of the effective stress when the point approaches the tool and strain rate drops almost to 0 . Then, when material begins slides along the tool, stress arises

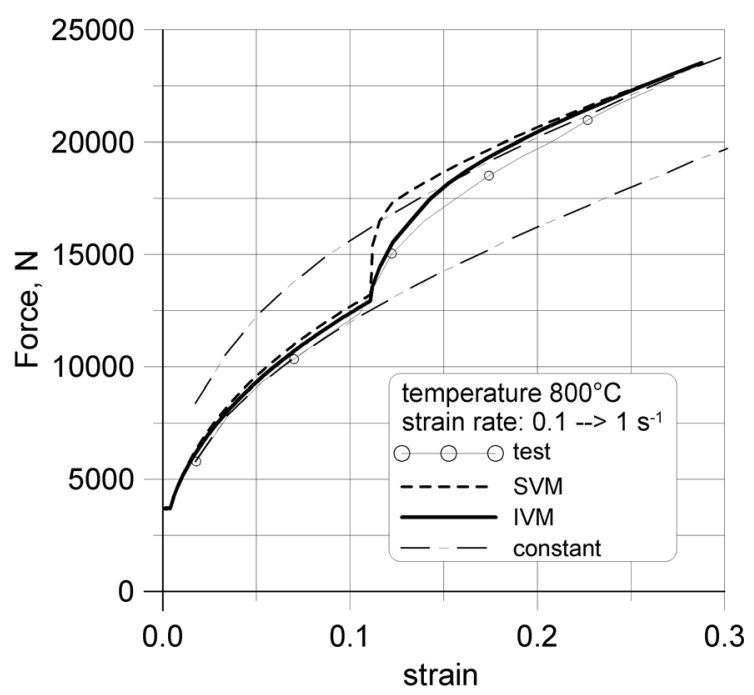

(b)

Fig. 4 Measured and calculated force during the deformation at the temperature of $800^{\circ} \mathrm{C}$ with decreasing (a) and increasing (b) strain rate 
Fig. 5 Flow stress of central point of specimen during the plastometric test at the temperature of $800{ }^{\circ} \mathrm{C}(\mathbf{a})$ and $900^{\circ} \mathrm{C}(\mathbf{b})$ with changes of strain rate and results of simulation by the original (SVM) and modified models (mSVM)

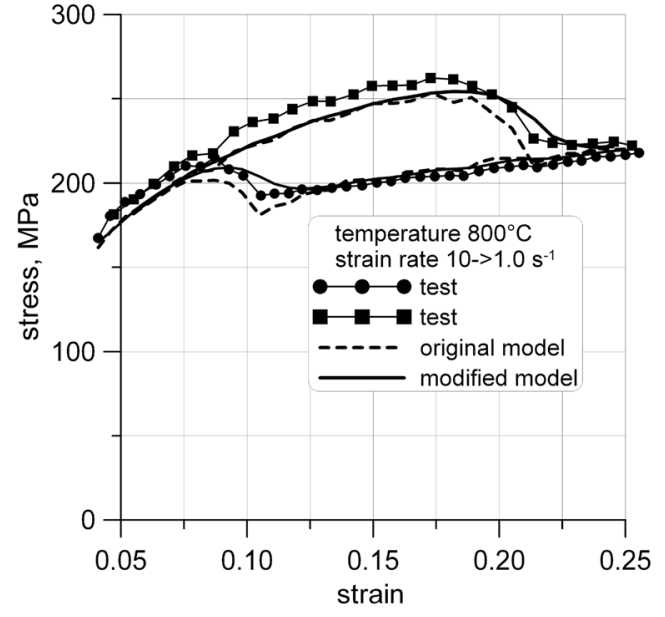

(a)

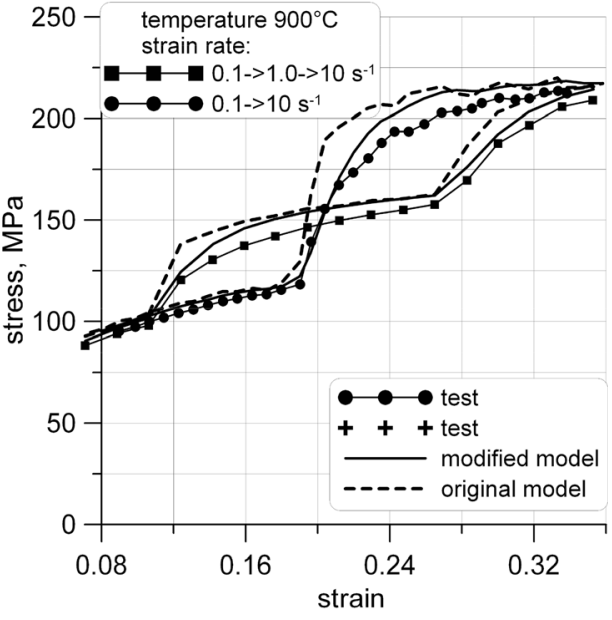

(b) again. $\mathrm{mSVM}$ for the same point at the same time shows only small reduction of the stress, because dynamic softening requires additional strain, which is very small because of low strain rate. The differences should affect the metal flow and the final shape.

The simulation results for the forging process confirmed that the developed model can be used for simulation not only of simple compression but also the more complex forming processes. Model can be also implemented into the commercial FEM codes. The IVM or mSVM can improve the accuracy of calculations and should be used for more precise modeling. However, those wishing to use the models in FEM simulations should remember that the models require additional variables in every element (or integration points) that require more memory and can limit the number of elements as well as increase the time required for calculations.
Fig. 6 Effective stress distribution during the forming (with control point locations)
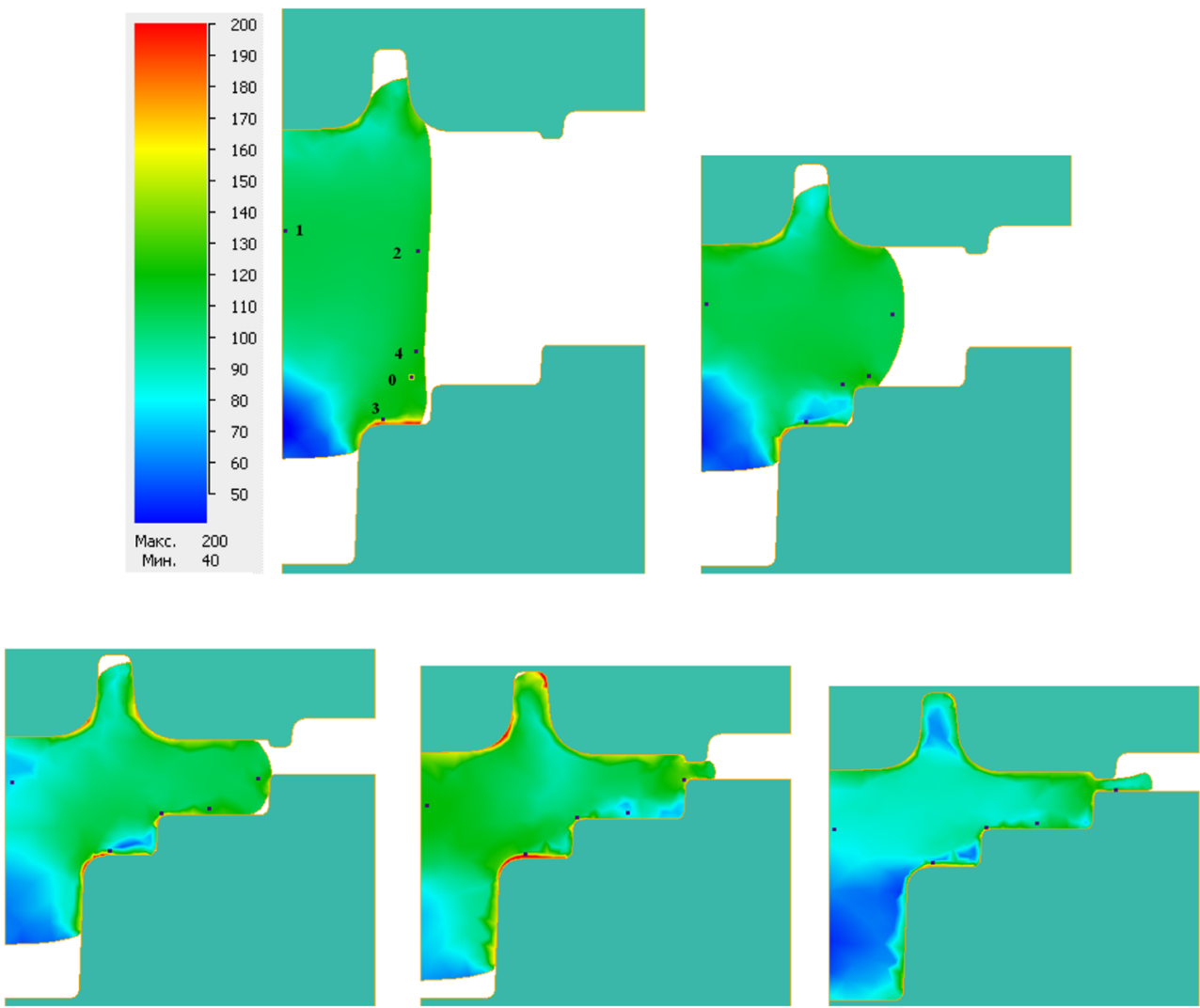


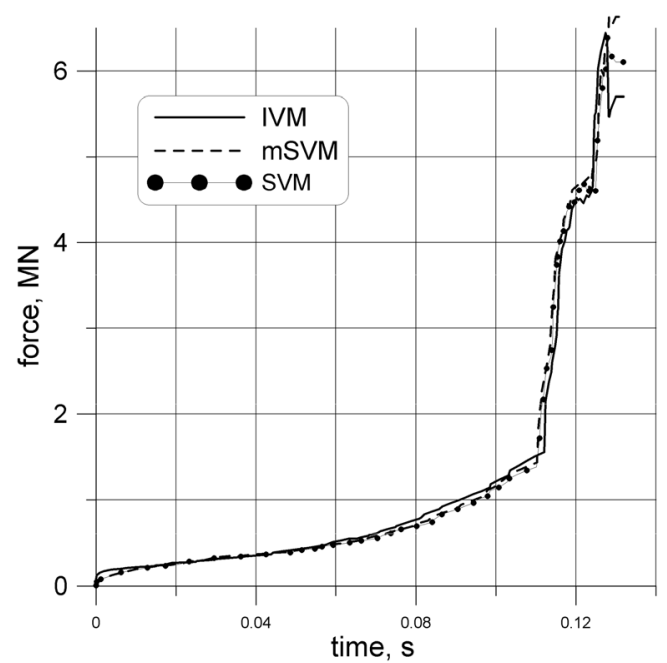

Fig. 7 Changes of compressive force during the forging

\section{Summary}

The effectiveness of internal variables modeled in varying deformation conditions is discussed in the paper. Different models and methods were implemented into different FEM codes for confirmation of their effectiveness. Internal variables models are represented by the original model [16] and by the modified state variables model, which receives properties of IVM with introduction of the three internal variables [14]. State variables models (SVM) are represented by the Sellars-Davenport model, table method with nonlinear interpolation and others. The effectiveness was evaluated by comparing results of FEM simulations with measurements carried out on thermomechanical simulator GLEEBLE 3800. Compressive force and flow stress were compared. Table with interpolation demonstrates the best results when constant deformation conditions are maintained, especially the same as in plastometric tests. But SVMs are unable to take into account varying deformation conditions properly. IVMs are more effective when deformation conditions are changing, especially very fast. The changes of the deformation conditions occur continuously in various areas of the deformed body and with different intensity. In such situations, only the internal variable model with proper reaction on changed conditions is able to predict the mechanical response of the deformed body.
Fig. 8 Changes of flow stress in control points

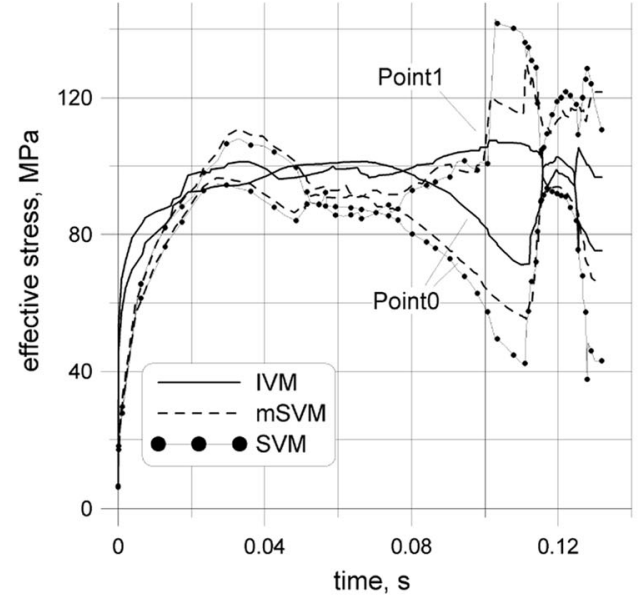

(a)

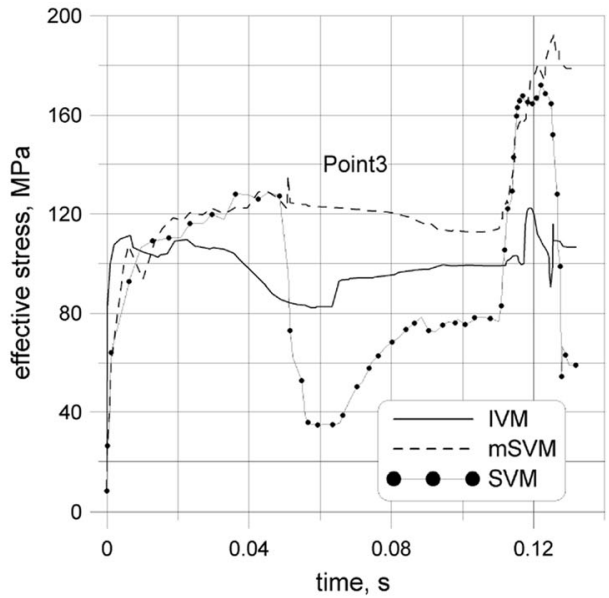

(b)

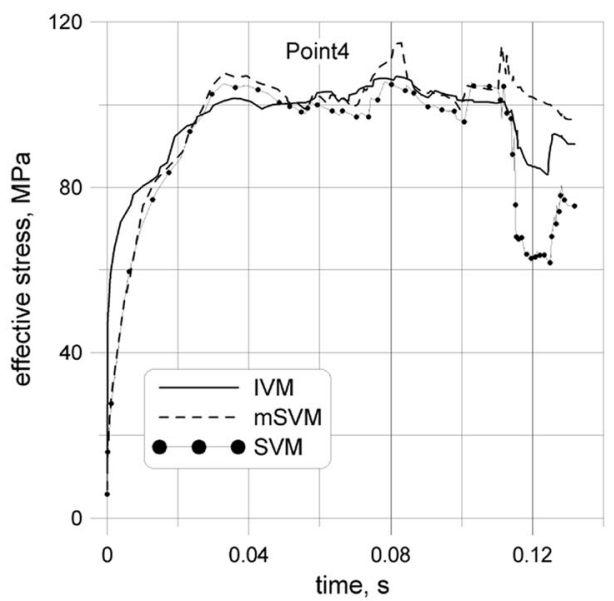

(c) 
Acknowledgments Financial support of the Polish Ministry of Science and Higher Education (grant no. N508 620 140) is greatly appreciated.

Open Access This article is distributed under the terms of the Creative Commons Attribution 4.0 International License (http:// creativecommons.org/licenses/by/4.0/), which permits unrestricted use, distribution, and reproduction in any medium, provided you give appropriate credit to the original author(s) and the source, provide a link to the Creative Commons license, and indicate if changes were made.

\section{References}

1. Kocks UF (1976) Laws for work-hardening and low-temperature creep. J Eng Mater Technol 98:76-85. doi:10.1115/1.3443340

2. Roberts W (1984) Dynamic changes that occur during hot working and their significance regarding microstructural development and hot workability. In: Krauss G (ed) Deformation, processing and structure, ASM, Metals Park OH, pp 109-184

3. Yoshie A, Morikawa H, Onoe Y (1987) Formulation of static recrystallization of austenite in hot rolling process of steel plate. Trans ISIJ 27:425-431

4. Bergström Y (1969/70) Dislocation model for the stress-strain behaviour of polycrystalline $\alpha$-Fe with special emphasis on the variation of the densities of mobile and immobile dislocations. Mater Sci Eng 5:193-200

5. Estrin Y, Mecking H (1984) A unified phenomenogical description of work hardening and creep based on one parameter models. Acta Metall 29:57-70. doi:10.1016/0001-6160(84)90202-5

6. Kocks UF, Mecking H (2003) Physics and phenomenology of strain hardening: the FCC case. Prog Mater Sci 48:171-273. doi: 10.1016/S0079-6425(02)00003-8

7. Estrin Y, Tóth LS, Molinari A, Bréchet Y (1998) A dislocationbased model for all hardening stages in large strain deformation. Acta Mater 46:5509-5522. doi:10.1016/S1359-6454(98)00196-7

8. Roters F, Raabe D, Gottstein G (2000) Work hardening in heterogeneous alloys - a microstructural approach based on three internal state variables. Acta Mater 48:4181-4189. doi:10.1016/S13596454(00)00289-5

9. Van Houtte P, Van Bael A, Seefeldt M (2007) The application of multiscale modelling for the prediction of plastic anisotropy and deformation textures. Mater Sci Forum 550:13-22. doi:10.4028/ www.scientific.net/MSF.550.13

10. Sandström R, Lagneborg R (1975) A model for hot working occurring by recrystallization. Acta Metall 23:387-398. doi:10.1016/ 0001-6160(75)90132-7

11. Roucoules C, Pietrzyk M, Hodgson PD (2003) Analysis of work hardening and recrystallization during the hot working of steel using a statistically based internal variable model. Mater Sci Eng A 339:1-9. doi:10.1016/S0921-5093(02)00120-X

12. Ordon J, Kuziak R, Pietrzyk M (2000) History dependent constitutive law for austenitic stainless steels. In: Pietrzyk M, Kusiak J, Majta J, Hartley P, Pillinger I (eds.) Proceedings of metal forming 2000, Krakow, pp 747-753

13. Estrin Y (1996) Dislocation density-related constitutive modeling. In: Krausz AS, Krausz K (eds) Unified constitutive law of plastic deformation. Academic, San Diego, pp 69-106
14. Svyetlichnyy DS (2005) The coupled model of a microstructure evolution and a flow stress based on the dislocation theory. ISIJ Int 45:1187-1193. doi:10.2355/isijinternational.45.1187

15. Svyetlichnyy DS (2009) Modification of coupled model of microstructure evolution and flow stress: experimental validation. Mater Sci Technol 25:981-988. doi:10.1179/174328408X317084

16. Svyetlichnyy DS, Majta J, Nowak J (2013) A flow stress for the deformation under varying condition - internal and state variable models. Mater Sci Eng A 576:140-148. doi:10.1016/j.msea.2013. 04.007

17. Taylor GI (1934) The mechanism of plastic deformation of crystals. Part I. Theoretical. Proc Roy Soc A 145:362-387. doi:10.1098/ rspa.1934.0106

18. Sellars CM (1990) Modelling microstructural development during hot rolling. J Mater Sci Technol 6:1072-1081. doi:10.1179/mst. 1990.6.11.1072

19. Qian M, Guo ZX (2004) Cellular automata simulation of microstructural evolution during dynamic recrystallization of an HY-100 steel. Mater Sci Eng A 365:180-185. doi:10.1016/j.msea.2003.09. 025

20. Svyetlichnyy DS (2010) Modelling of the microstructure: from classical cellular automata approach to the frontal one. Comput Mater Sci 50:92-97. doi:10.1016/j.commatsci.2010.07.011

21. Svyetlichnyy DS (2012) Simulation of microstructure evolution during shape rolling with the use of frontal cellular automata. ISIJ Int 52:559-568. doi:10.2355/isijinternational.52.559

22. Svyetlichnyy DS (2012) Reorganization of cellular space during the modeling of the microstructure evolution by frontal cellular automata. Comput Mater Sci 60:153-162. doi:10.1016/j.commatsci. 2012.03.029

23. Davenport SB, Silk NJ, Sparks CN, Sellars CM (2000) Development of constitutive equations for modelling of hot rolling. Mater Sci Technol 16:539-546. doi:10.1179/ 026708300101508045

24. Sellars CM, Tegart WJ, Mc G (1966) La relation entre la resistance et la structure dans la deformation a chaud. Mem Sci Rev Metall 63: 731-746

25. Malinowski Z (2001) Analysis of temperature fields in the tools during forging of axially symmetrical parts. Arch Metall 46:93-118

26. Malinowski Z, Rywotycki M (2009) Modeling of the strand and mold temperature in the continuous steel caster. Arch Civ Mech Eng 9:59-73. doi:10.1016/S1644-9665(12)60060-0

27. Bíró T, Csizmadia J (2012) Recent technique for thermal-fatigue simulation of heat-resistant steels. Mech Eng 56:105-110. doi:10. 3311/pp.me.2012-2.05

28. Shida S (1968) Effect of carbon content, temperature and strain rate on compressive flow-stress of carbon steel (Resistance to deformation of carbon steels at elevated temperature, 1st report). J JSTP 9: 127-132

29. Perig AV, Golodenko NN (2014) CFD 2D simulation of viscous flow during ECAE through a rectangular die with parallel slants. Int J Adv Manuf Technol 74:943-962. doi:10.1007/s00170-014-5827-2

30. Stebunov S, Biba N, Ovchinnikov A, Smelev V (2007) Application of QForm forging simulation system for prediction of microstructure of aluminium forged parts. Comp Meth Mater Sci 7:1-5 